\title{
Latest Results from The Olympus Experiment
}

\author{
Axel Schmidt*† \\ Massachusetts Institute of Technology \\ E-mail: schmidta@mit.edu
}

\begin{abstract}
The two experimental techniques for determining the proton's elastic form factors-unpolarized cross section measurements and polarization asymmetries-have yielded strikingly discrepant results. One possible explanation for this discrepancy is that hard two-photon exchange, a radiative correction that is typically neglected, contributes on the percent level to the elastic electron-proton cross section. The OLYMPUS experiment tests this hypothesis by measuring the ratio of positronproton to electron-proton elastic scattering cross sections. Deviations in this ratio from unity are a signature of hard two-photon exchange.

The OLYMPUS experiment took place at DESY, in Hamburg, Germany, and over $4 \mathrm{fb}^{-1}$ of integrated luminosity were acquired by the end of data taking in 2013. $2 \mathrm{GeV}$ electron and positron beams, alternating daily, were directed through a windowless hydrogen gas target. Scattered leptons and recoiling protons were detected in coincidence in a toroidal magnetic spectrometer simultaneously over a wide range of angles. The relative luminosity between electron and positron modes was monitored redundantly through rates of both forward elastic and symmetric Møller/Bhabha scattering.
\end{abstract}

The latest results from the OLYMPUS analysis will be presented.

The 26th International Nuclear Physics Conference

11-16 September, 2016

Adelaide, Australia

${ }^{*}$ Speaker.

${ }^{\dagger}$ for the OLYMPUS Collaboration 


\section{Introduction}

Over the past few decades, a discrepancy has emerged between the proton's form factor ratio extracted from polarization asymmetries [1-6], and that same ratio extracted from unpolarized elastic electron-proton cross section measurements [7-12] (shown in figure 1). The proton's form factors, $G_{E}\left(Q^{2}\right)$ and $G_{M}\left(Q^{2}\right)$, encode the distributions of electromagnetic charge and current within the proton as functions of momentum transfer, $Q^{2}$. They should be universal properties of the proton. A discrepancy between measurement techniques suggests that one or both of the techniques is failing to account for some additional effect, distorting the results. Until this discrepancy is understood, there will be lingering uncertainty about the true values of the form factors.

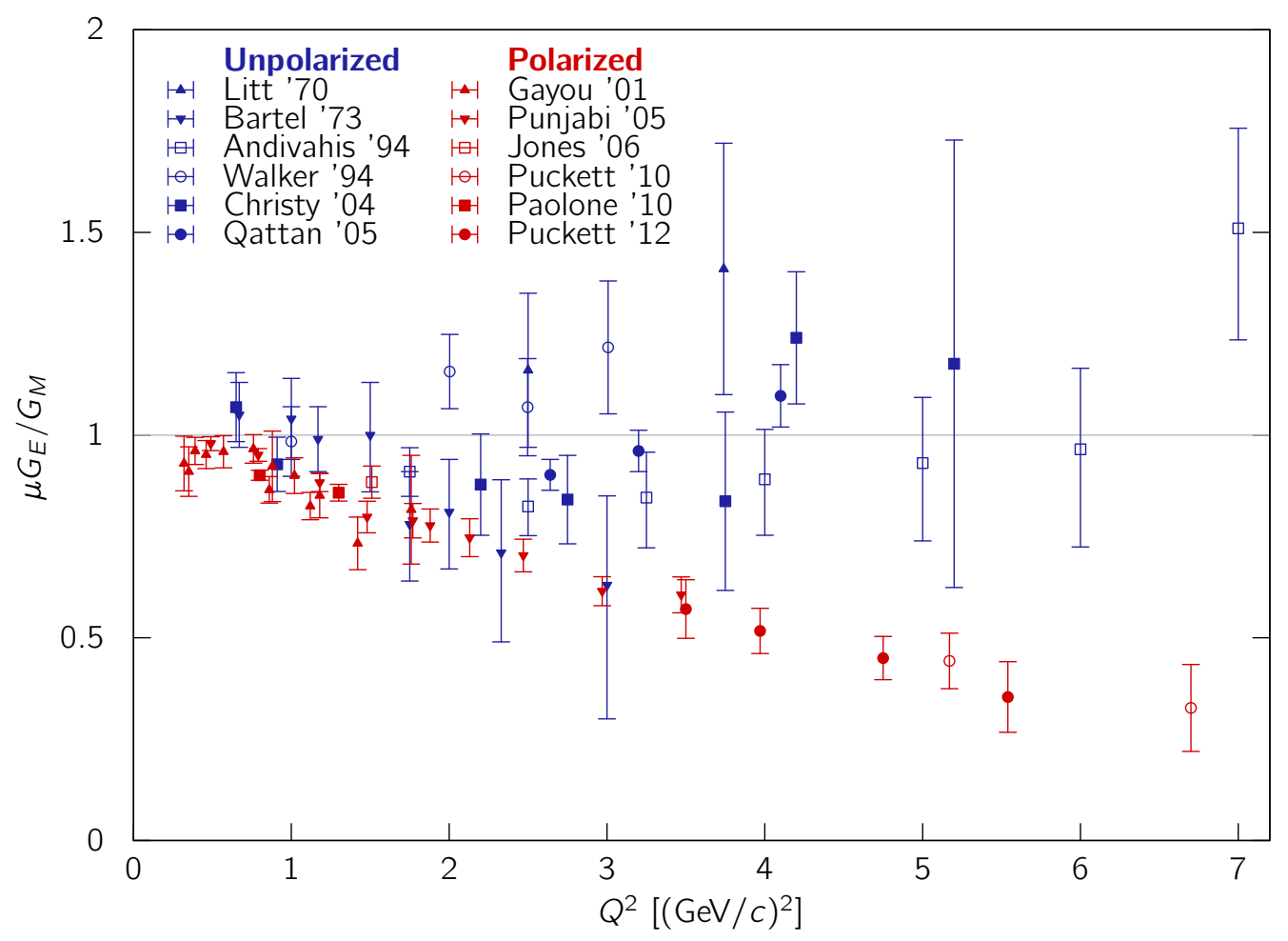

Figure 1: The proton's form factors as measured by polarization asymmetries (red) are inconsistent with those extracted from unpolarized cross sections (blue). Data are taken from [1-12].

One hypothesis for the cause of this discrepancy is that there is a non-negligible contribution to the elastic electron-proton scattering cross section from hard two-photon exchange (TPE) [13, 14]. Hard TPE is a radiative correction that is neglected in standard radiative correction prescriptions $[15,16]$. Some theoretical predictions suggest that a hard TPE contribution of only a few percent to the total cross section, provided that it has the correct kinematic dependence, would produce a discrepancy consistent with that of the measurements [17, 18].

The OLYMPUS experiment [19] tested this hypothesis by determining the hard TPE contribution to the elastic electron-proton scattering cross section from a measurement of the positronproton to electron-proton elastic cross section ratio. The results indicate a smaller hard TPE contribution than several theories predicted and are mostly consistent with no hard TPE effect [20]. 
However, phenomenological predictions show that a small TPE effect over the OLYMPUS kinematic range is consistent with the observed form factor discrepancy.

\section{Motivation}

Hard two-photon exchange is a plausible explanation for the proton form factor discrepancy. Standard radiative corrections prescriptions for elastic electron-proton scattering only account for TPE in the soft limit, in which one of the exchanged photons carries negligible momentum. Furthermore, hard TPE would affect the two form factor measurement techniques differently. The polarization asymmetry technique is mostly insensitive to a hard TPE contribution, while the Rosenbluth separation technique for determining $G_{E}^{2}$ and $G_{M}^{2}$ from unpolarized cross sections would be sensitive, especially at large $Q^{2}$.

Hard TPE is difficult to calculate, however, and no model-independent calculation has yet been produced. The challenge comes from the off-shell hadronic propagator. The proton can propagate as proton, but can also propagate as any excited state of the proton, as a $\Delta^{+}$for instance, and each excited state contributes to the amplitude. Several model dependent calculations have been made using a variety of techniques. Many suggest that TPE is the cause of the form factor discrepancy $[17,18,21]$, and some do not [22, 23]. Experimental verification is needed.

The experimental signature of hard TPE is a deviation from unity in the ratio of positron-proton to electron-proton elastic cross sections:

$$
R_{2 \gamma} \equiv \frac{\sigma_{e^{+} p \rightarrow e^{+} p}}{\sigma_{e^{-} p \rightarrow e^{-}}} .
$$

This ratio is unity at leading order. The next-to-leading order term is an interference between oneand two-photon exchange. This interference has opposite sign between electron scattering and positron scattering, so that the ratio amplifies this interference:

$$
R_{2 \gamma}=1+\frac{4 \operatorname{Re}\left[\mathscr{M}_{2 \gamma} \mathscr{M}_{1 \gamma}\right]}{\left|\mathscr{M}_{1 \gamma}\right|^{2}}+\mathscr{O}\left(\alpha^{4}\right) .
$$

Phenomenological predictions suggest that in order to resolve the discrepancy $R_{2 \gamma}$ must increase to several percent above unity, i.e., the positron cross section must increase relative to the electron cross section, as $\varepsilon$, the virtual photon polarization parameter given by $\varepsilon \equiv\left[1+2\left(1+\frac{Q^{2}}{4 m_{p}^{2}}\right) \tan ^{2} \frac{\theta_{e}}{2}\right]^{-1}$, decreases [24-26]. A ratio of $R_{2 \gamma}$ that remains at unity indicates that there is no contribution from hard TPE to the elastic cross section.

\section{Experiment}

The OLYMPUS experiment was conducted at DESY, in Hamburg, Germany, with data collection concluding in 2013. The DORIS storage ring provided a $2 \mathrm{GeV}$ stored beam of either electrons or positrons, with a switch between beam species occurring once per day. The beams passed through a windowless hydrogen gas target that was internal to the storage ring vacuum [27]. The typical luminosity was $2 \times 10^{33} \mathrm{~cm}^{-2} \mathrm{~s}^{-1}$, and approximately $4.5 \mathrm{fb}^{-1}$ were collected.

Surrounding the target was a large-acceptance toroidal magnetic spectrometer, which was formerly used in the BLAST experiment at MIT-Bates [28]. A schematic is shown in figure 2. The 


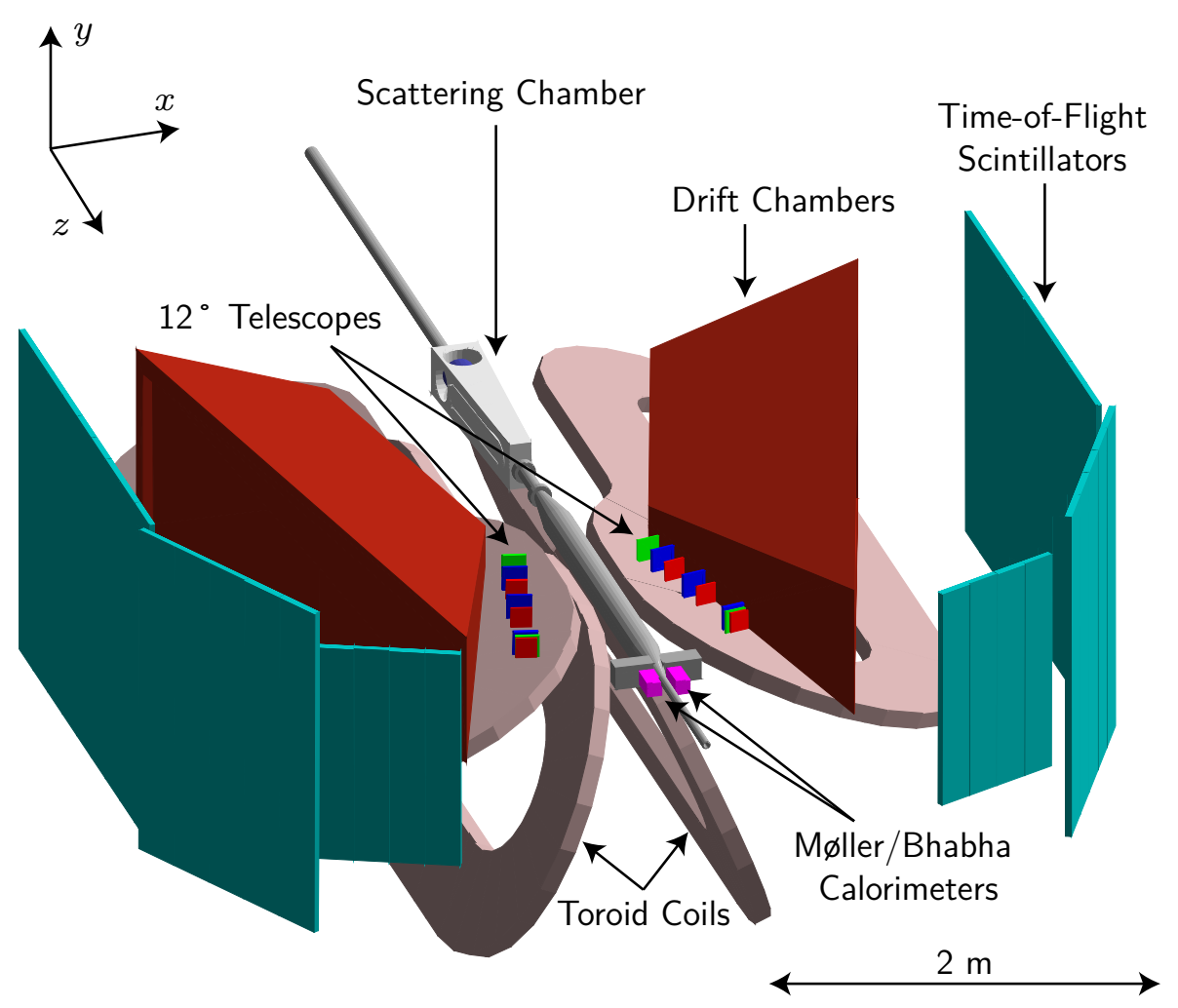

Figure 2: A schematic of the OLYMPUS spectrometer is shown without the top four toroid magnet coils. The beam enters from the upper left.

spectrometer had two identical instrumented sectors to allow the detection of a scattered lepton in coincidence with a recoiling proton. Drift chambers were the main tracking detectors, while a wall of scintillator bars, called the Time-of-Flight system, were used for triggering and timing.

Since the measurement of $R_{2 \gamma}$ relied on an accurate determination of the relative luminosity between the positron and electron running modes, three new systems were developed to monitor luminosity. The "slow control" system was used to make an estimate online from measurements of the beam current and target flow. Two forward tracking telescopes installed at the $12^{\circ}$ scattering angle monitored luminosity through the rate of forward $e p$ scattering. A pair of calorimeters installed at $1.3^{\circ}$, monitored the rate of Møller/Bhabha scattering from atomic electrons in the hydrogen target [29].

\section{Results}

The OLYMPUS results [20] are shown in figure 3. The smaller error bars indicate statistical uncertainty, while the larger error bars indicate the quadratic sum of statistical and point-to-point uncorrelated systematic uncertainties. The gray band below the data indicates the size of the correlated systematic uncertainty, though the correlations between data points are non-trivial and are not well-described by a simple scale factor. The data are not inconsistent with unity, suggesting that hard TPE has a small effect on the elastic cross section. 


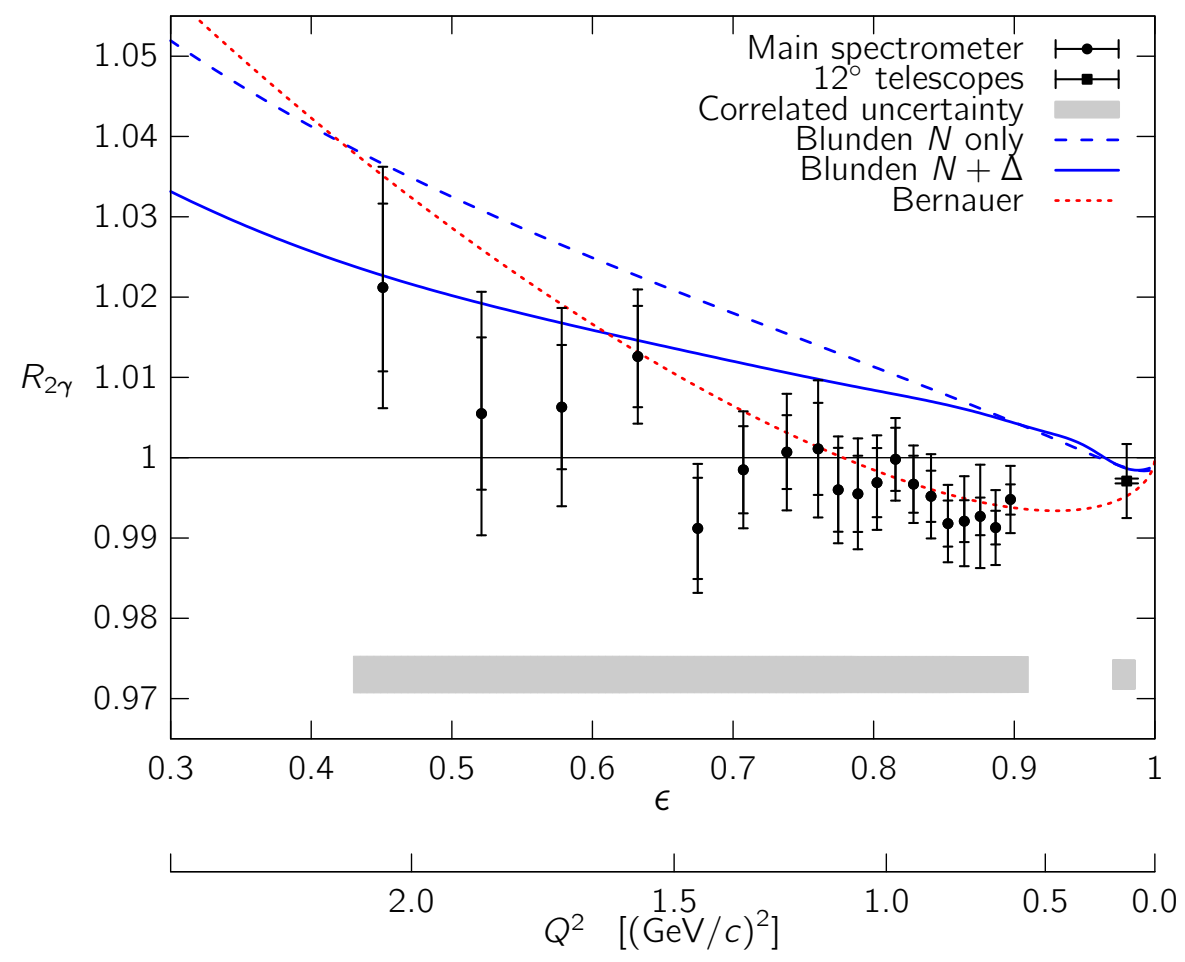

Figure 3: The OLYMPUS results are smaller than the theory calculation of Blunden, but consistent with the phenomenological prediction of Bernauer.

In addition to the data, the theory calculation of hard two photon exchange by P. G. Blunden [30] is shown; the blue solid line shows the calculation of the TPE diagram assuming only a nucleon propagator, while the blue dashed line assumes that both the nucleon and $\Delta$-resonance contribute to the propagator. The data sit below this theory calculation, suggesting that some additional terms or corrections are needed to effectively describe hard TPE.

The red dotted curve shows a phenomenological prediction by J. C. Bernauer et al. [26], which is made by fitting an ansatz for the functional form of the hard TPE contribution to both polarized and unpolarized data. In essence, it describes how large of a TPE effect is necessary to resolve the proton form factor discrepancy. This prediction does a reasonable job of matching the OLYMPUS data, indicating that the TPE hypothesis is still viable.

\subsection{Comparison with CLAS and VEPP-3}

OLYMPUS was one of three contemporary experiments to measure $R_{2 \gamma}$. In addition to OLYMPUS, a measurement using alternating $e^{+}$and $e^{-}$beams was made at the VEPP-3 storage ring in Novosibirsk, Russia [31], and a measurement using a tertiary beam of $e^{+} / e^{-}$pairs was made by the CLAS Collaboration at Jefferson Lab, in the United States [32, 33]. The kinematic coverage of the three experiments is shown in figure 4. CLAS released results with two different binning schemes; figure 4 shows the points using their constant $Q^{2}$ scheme, plus four non-overlapping bins from their constant $\varepsilon$ scheme at low $Q^{2}$. 


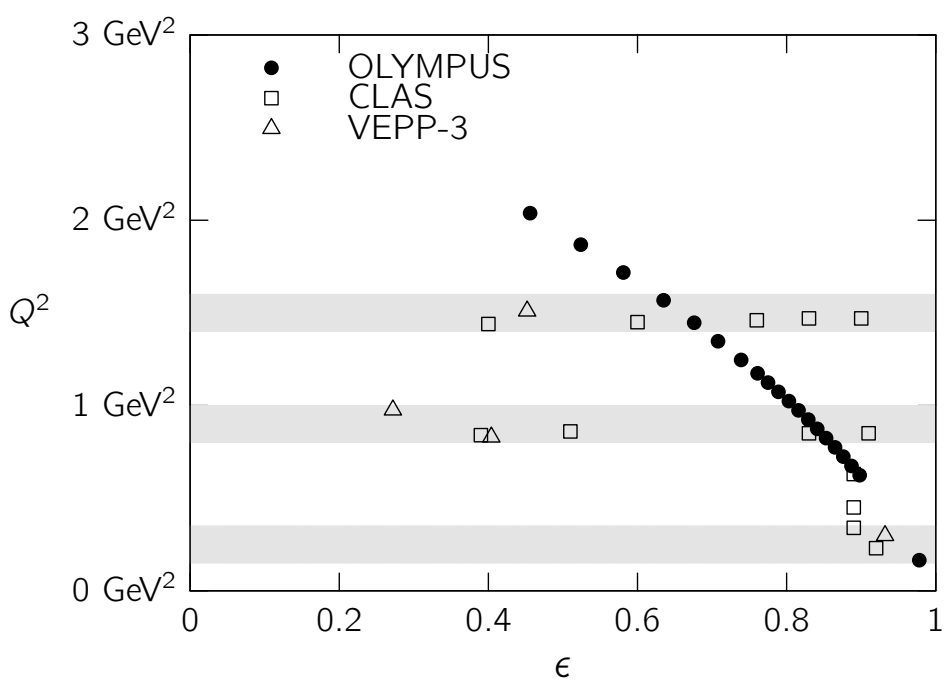

Figure 4: Comparing the results of the three contemporary TPE experiments is not straight-forward because they cover slightly different kinematic points. Figure 5 shows the results as a function of $\varepsilon$ for three different $Q^{2}$ values, shown here as shaded bands.

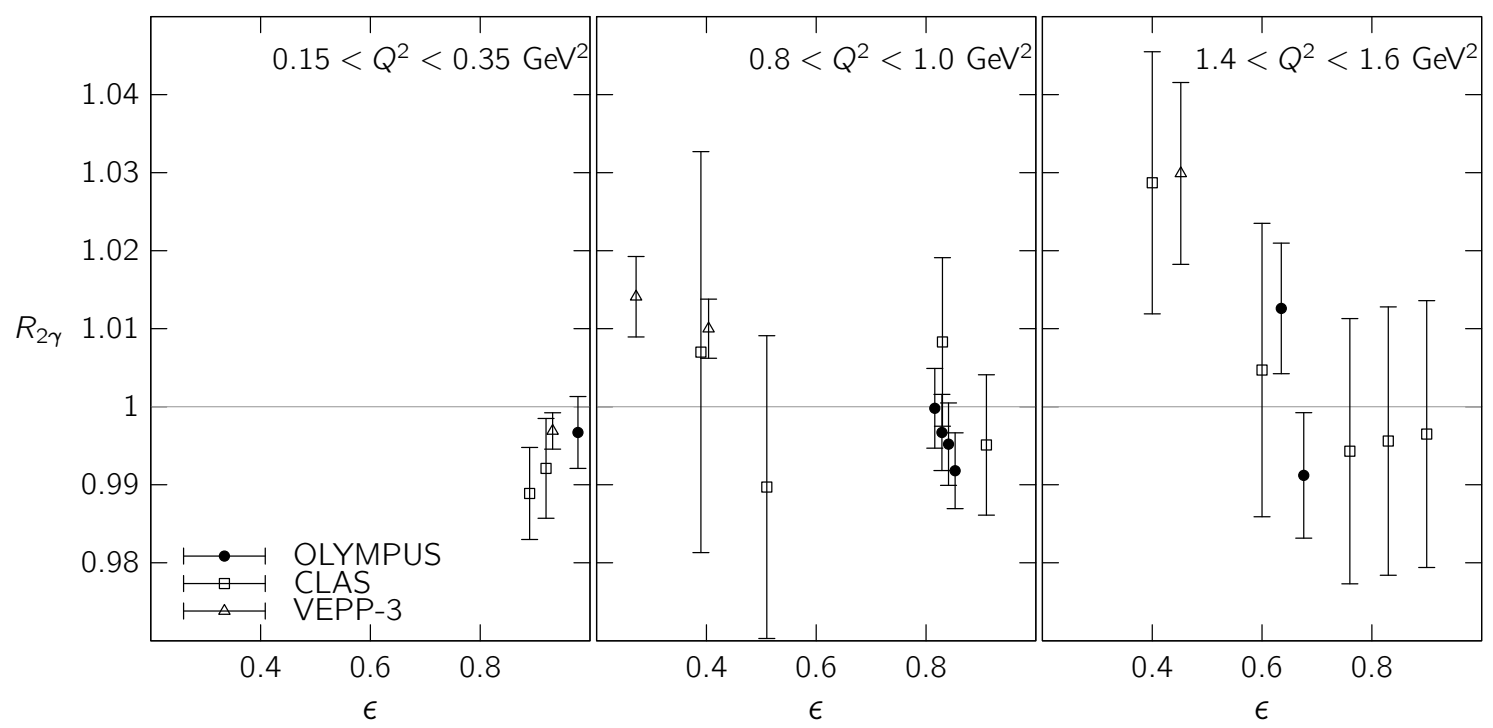

Figure 5: The results of the three contemporary experiments, shown for three different $Q^{2}$ ranges, are largely consistent with each other.

Figure 5 compares the results of the three contemporary experiments. Since OLYMPUS, CLAS, and VEPP-3 cover slightly different kinematic points, the results are shown for three different ranges in $Q^{2}$, highlighted in figure 4 by three shaded bands. The error bars are the quadratic sum of statistical and uncorrelated point-to-point systematic errors. The published VEPP-3 results are relative to the value of $R_{2 \gamma}$ at one of two high- $\varepsilon$ "luminosity normalization points" (LNPs). These LNPs are at a similar kinematic point to the OLYMPUS highest $\varepsilon$ point, and figure 5 uses the OLYMPUS measurement at that point to normalize the VEPP- 3 results. The results of the three 
experiments are largely consistent with each other and show that, over the covered kinematic range, $R_{2 \gamma}$ is less than $3 \%$ above unity.

\section{Conclusions}

The OLYMPUS experiment has made the highest precision measurement of $R_{2 \gamma}$ to date over a wide range of $\varepsilon$ and $Q^{2}$. OLYMPUS measured $R_{2 \gamma}$ to be smaller than Blunden's calculation, especially at high $\varepsilon$ and low $Q^{2}$. In fact, the results are largely consistent with unity, which would indicate no hard TPE. This does not, however, eliminate the TPE hypothesis for the solution to the proton form factor discrepancy. The OLYMPUS data are also consistent with the phenomenological prediction by Bernauer et al., suggesting that OLYMPUS did not probe kinematics where significant TPE was needed to solve the discrepancy.

A more conclusive test would be a measurement at higher $Q^{2}$ where the form factor discrepancy is larger and where the $R_{2 \gamma}$ is predicted to be greater. Such an experiment would be difficult because the elastic scattering cross section drops at high $Q^{2}$. However such a measurement would be the clearest experimental verification that hard TPE is responsible for the discrepancy in the proton's form factors.

\section{References}

[1] O. Gayou et al., Measurements of the elastic electromagnetic form factor ratio $\mu_{p} G_{E_{p}} / G_{M_{p}}$ via polarization transfer, Phys. Rev. C 64, 038202, 2001

[2] V. Punjabi et al., Proton elastic form factor ratios to $Q^{2}=3.5 \mathrm{GeV}^{2}$ by polarization transfer Phys. Rev. C 71, 055202, 2005

[3] M. K. Jones et al., Proton $G_{E} / G_{M}$ from beam-target asymmetry Phys. Rev. C 74, 035201, 2006

[4] A. J. R. Puckett et al., Recoil Polarization Measurements of the Proton Electromagnetic Form Factor Ratio to $Q^{2}=8.5 \mathrm{GeV}^{2}$, Phys. Rev. Lett. 104, 242301, 2010

[5] M. Paolone et al., Polarization Transfer in the ${ }^{4} \mathrm{He}\left(\vec{e}, e^{\prime} \vec{p}\right)^{3} \mathrm{H}$ Reaction at $Q^{2}=0.8$ and $1.3(\mathrm{GeV} / \mathrm{c})^{2}$, Phys. Rev. Lett. 105, 072001, 2010

[6] A. J. R. Puckett et al., Final analysis of proton form factor ratio data at $Q^{2}=4.0,4.8$, and 5.6 GeV ${ }^{2}$, Phys. Rev. C 85, 045203, 2012

[7] J. Litt et al., Measurement of the ratio of the proton form factors $G_{E} / G_{M}$ at high momentum transfers and the question of scaling, Phys. Lett. B 31 40-44, 1970

[8] W. Bartel et al., Measurement of proton and neutron electromagnetic form factors at squared four-momentum transfers up to $3(\mathrm{GeV} / \mathrm{c})^{2}$, Nucl. Phys. B 58, 429-475, 1973

[9] L. Andivahis et al., Measurements of the electric and magnetic form factors of the proton from $Q^{2}=1.25$ to $8.83(\mathrm{Gev} / \mathrm{c})^{2}$, Phys. Rev. D 50, 5491-5517, 1994 
[10] R. C. Walker et al., Measurements of the proton elastic form factors for $1 \leq Q^{2} \leq 3(\mathrm{GeV} / \mathrm{c})^{2}$ at SLAC, Phys. Rev. D 49, 5671-5689, 1994

[11] M. E. Christy et al., Measurements of electron-proton elastic cross sections for $0.4<Q^{2}<$ $5.5(\mathrm{GeV} / \mathrm{c})^{2}$, Phys. Rev. C 70, 015206, 2004

[12] I. A. Qattan et al., Precision Rosenbluth Measurement of the Proton Elastic Form Factors, Phys. Rev. Lett. 94, 142301, 2005

[13] P. A. M. Guichon, M. Vanderhaeghen, How to Reconcile the Rosenbluth and the Polarization Transfer Methods in the Measurement of the Proton Form Factors, Phys. Rev. Lett. 91, 142303-1, 2003

[14] P. G. Blunden et al., Two-Photon Exchange and Elastic Electron-Proton Scattering Phys. Rev. Lett. 91, 142304, 2003

[15] L. W. Mo, Y. S. Tsai, Radiative Corrections to Elastic and Inelastic ep and $v p$ Scattering, Rev. Mod. Phys. 41, 205-235, 1969

[16] L. C. Maximon, J. A. Tjon, Radiative corrections to electron-proton scattering Phys. Rev. C 62, 054320, 2000

[17] A. V. Afanasev et al., Two-photon exchange contribution to elastic electron-nucleon scattering at large momentum transfer, Phys. Rev. D 72, 013008, 2005

[18] P. G. Blunden et al., Two-photon exchange in elastic electron-nucleon scattering, Phys. Rev. C 72, 034612, 2005

[19] R. Milner et al., The OLYMPUS Experiment, Nucl. Instr. Meth. A741 1-17, 2014

[20] B. S. Henderson et al., Hard two-photon contribution to elastic lepton-proton scattering determined by the OLYMPUS experiment, Phys. Rev. Lett., Accepted December 23, 2016

[21] N. Kivel, M. Vanderhaeghen, Two-Photon Exchange in Elastic Electron-Proton Scattering: A QCD Factorization Approach Phys. Rev. Lett. 104, 092004, 2009

[22] Y. M. Bystritskiy et al., Structure function method applied to polarized and unpolarized electron-proton scattering: A solution of the $G_{E}(p) / G_{M}(p)$ discrepancy, Phys. Rev. $C 75$ 015207, 2007

[23] E. A. Kuraev et al., Charge asymmetry for electron (positron)-proton elastic scattering at large angles Phys. Rev. C 78, 015205, 2008

[24] Y. C. Chen et al., Is there model-independent evidence of the two-photon-exchange effect in the electron-proton elastic scattering cross section?, Phys. Lett. B 652, 269-274, 2007

[25] J. Guttmann et al., Determination of two-photon exchange amplitudes from elastic electronproton scattering data, Eur. Phys. J. A 47, 77, 2011 
[26] J. C. Bernauer et al., The electric and magnetic form factors of the proton, Phys. Rev. C 90, 015206, 2014

[27] J. C. Bernauer et al., The OLYMPUS internal hydrogen target, Nucl. Instr. Meth. A 755, 20-27, 2014

[28] D. K. Hasell et al., The BLAST Experiment, Nucl. Instr. Meth. A 603, 247-262, 2009

[29] R. Perez Benito et al., Design and performance of a lead fluoride detector as a luminosity monitor, Nucl. Instr. Meth. A 826, 6-14, 2016

[30] P. G. Blunden et al., Private communication, 2016

[31] I. A. Rachek et al., Measurement of the Two-Photon Exchange Contribution to the Elastic $e^{ \pm} p$ Scattering Cross Sections at the VEPP-3 Storage Ring, Phys. Rev. Lett 114, 0062005, 2015

[32] D. Adikaram et al., Towards a Resolution of the Proton Form Factor Problem: New Electron and Positron Scattering Data, Phys. Rev. Lett. 114, 062003, 2015

[33] D. Rimal et al., Measurement of two-photon exchange effect by comparing elastic $e^{ \pm} p$ cross sections, [nucl-ex/1603.00315], 2016 